\title{
DIMENSIONAL REDUCTION OF THE FOKKER-PLANCK EQUATION FOR STOCHASTIC CHEMICAL REACTIONS
}

\author{
PER LÖTSTEDT ${ }^{1}$, LARS FERM $^{1}$ \\ ${ }^{1}$ Div of Scientific Computing, Dept of Information Technology \\ Uppsala University, SE-75105 Uppsala, Sweden \\ emails: perl, ferm @it.uu.se
}

\begin{abstract}
The Fokker-Planck equation models chemical reactions on a mesoscale. The solution is a probability density function for the copy number of the different molecules. The number of dimensions of the problem can be large making numerical simulation of the reactions computationally intractable. The number of dimensions is reduced here by deriving partial differential equations for the first moments of some of the species and coupling them to a Fokker-Planck equation for the remaining species. With more simplifying assumptions, another system of equations is derived consisting of integro-differential equations and a Fokker-Planck equation. In this way, the simulation of the chemical networks is possible without the exponential growth in computatational work and memory of the original equation and with better modelling accuracy than the macroscopic reaction rate equations. Some terms in the equations are small and are ignored. Conditions are given for the influence of these terms to be small on the equations and the solutions. The difference between different models is illustrated in a numerical example.
\end{abstract}

Keywords: master equation, Fokker-Planck equation, reaction rate equations, dimensional reduction

AMS subject classification: 65M20, 65M50

\section{Introduction}

Simulation in silico of biological cells is important in the emerging field of systems biology [8, 18]. In computer simulations, the models of the complicated regulatory networks in a cell can be compared quantitatively to experimental 
data. The macroscopic model equations for simulation of biochemical reactions in the cells are the reaction rate equations. They form a system of deterministic, nonlinear ordinary differential equations (ODEs) for the concentrations of the participating chemical species. This model is accurate for reactions with many participating molecules of each species. If the number of molecules is small or if the reactions occur far from thermodynamic equilibrium, then a stochastic model on a mesoscale such as the master equation $[2,9,10,16,24]$ is a much more precise description. This is often the case in molecular biology where the majority of the reacting molecules are found in copy numbers less than one hundred in cells such as Escherichia coli [14]. An example where a macroscopic model is insufficient and a stochastic view is necessary is found in [25] where a biological clock is investigated.

The master equation $[12,16]$ is a differential-difference equation for the time dependent probability density function (PDF) $p$ of the number of molecules of each species. The dimension of the discrete spatial domain of $p$ is the same as the number of species. Since there are tens of thousands of active proteins in a cell, the size of the computational problem to solve the equation can be formidable. The Fokker-Planck (FP) equation is a partial differential equation (PDE) for $p$ in time and continuous space approximating the master equation [12, 16, 23]. The spatial dimension is the same as in the master equation. The FP equation can be solved numerically after discretization using techniques for PDEs, but for many molecular species and many spatial dimensions other methods are needed due to the exponential growth of computational work and storage requirements with increasing dimension ('the curse of dimensionality'). There are deterministic and stochastic algorithms tailored for high dimensional problems. Sparse grids is a deterministic method designed for high dimensional problems [3]. Stochastic methods e.g. for high-dimensional integrals [4] and Gillespie's stochastic simulation algorithm (SSA) [13] for the master equation do not suffer from the curse of dimensionality. Gillespie's method is a Monte Carlo method simulating the reactions step by step and is the standard method to simulate mesoscopic biochemical reactions in cells.

The dimensionality of the problem is reduced in this paper by assuming that certain species are normally distributed with a small variance. Then a system of PDEs is derived, similar to the FP equation, for a quantity proportional to the expected values of the species. The PDF of the remaining species satisfies the FP equation. Suppose that $m$ species are handled in the ususal way with the FP equation, $n$ species are treated in the special way approximated by a normal distribution, and that $L$ grid points are needed in each coordinate direction to resolve the FP equation. Then the original problem with $N=m+n$ species requires $L^{m+n}$ grid points and variables for a representation of the PDF with the FP equation. The reduced problem is solved in $m$ dimensions with $n+1$ unknowns thus needing only $(n+1) L^{m}$ memory cells to store the solution. The quotient between the storage requirements of the two methods is $(n+1) / L^{n}$, a substantial 
improvement when $L>1$ and $n$ is large. Assuming that the expected values in the normal distribution are independent of space, an intermediate model is obtained with integro-differential equations (IDEs) for the expected values coupled to the FP equation. Some species should be modelled with full generality with the FP equation, e.g. if they are present in small copy numbers, while other species are well approximated by a normal distribution with a small variance, e.g. if there are many molecules of this kind. If $m=0$, then we recover the macroscopic reaction rate equations and if $n=0$, then we have the full mesoscopic FP equation. The simplification of the FP equation is motivated by applications in molecular biology but is applicable also to other problems governed by different forms of the FP equation.

A similar partitioning of the variables is made in $[5,15,17,21]$ to improve the efficiency of the SSA algorithm. The species are either fast or slow and the fast variables satisfy ODEs and the slow variables are simulated by the SSA method $[15,17]$. The deterministic part and the stochastic part are coupled in the same manner as the IDEs and the FP equation.

The same reduction of the computational work is achieved in the quantumclassical molecular dynamics (QCMD) model for simulation of the time evolution of large molecules. Each degree of freedom of the molecule introduces one dimension in the Schrödinger equation and quickly makes it impossible to compute the solution for molecules with more than a few atoms. Therefore, most atoms are described by classical mechanics while a critical small part of the system is treated with quantum mechanics. See e.g. [6, 7] for numerical schemes suitable for this approximation.

The paper is organized as follows. The reaction rate equations, the master equation and the FP equation are given in the next section. Different ways of approximating the FP equation in fewer dimensions are introduced in Section 3. Some of the molecular species are assumed to be normally distributed with a small variance. Equations are derived for the expected values of these species and a FP equation for the remaining species. If all species have a normal distribution, then we obtain the macroscopic reaction rate equations. In Section 4, three different simplifications of the original FP equation are compared in a numerical example with four chemical compounds.

The $i$ :th element of a vector $\mathbf{v}$ is denoted by $v_{i}$. If $\mathbf{v}$ has the length $N$ then the $\ell_{2}$-norm is $\|\mathbf{v}\|_{2}=\left(\sum_{i=1}^{N}\left|v_{i}\right|^{2}\right)^{1 / 2}$ in the paper.

\section{The reaction rate, master and Fokker-Planck equations}

In a spatially homogeneous mixture of molecules, biochemical reactions in a cell are modeled on a macroscale by the reaction rate equations. They form a system 
of nonlinear ODEs for the concentrations of the molecular species. A stochastic model on the mesoscale is governed by the master equation [16]. Its solution is the PDF for the copy numbers of the particpating molecules. The FP equation is obtained after Taylor expansion in space of the master equation.

Assume that there are $N$ chemically active molecular species $X_{i}, i=1 \ldots N$, and that there are $x_{i}$ molecules of substrate $X_{i}$ at time $t$. Then $x_{i}$ is a nonnegative integer number, $x_{i} \in \mathbb{Z}_{+}$, and is a component in the state vector $\mathbf{x}$. A chemical reaction $r$ is a transition from a state $\mathbf{x}_{r}$ to $\mathbf{x}$ so that $\mathbf{x}_{r}=\mathbf{x}+\mathbf{n}_{r}$. The non-negative propensity $w_{r}\left(\mathbf{x}_{r}, t\right)$ is the probability for the reaction to occur per unit time. It is often modeled as a polynomial or in a Michaelis-Menten model it is a rational polynomial in $x_{i}$. The reaction $r$ can now be written

$$
\mathbf{x}_{r} \stackrel{w_{r}\left(\mathbf{x}_{r}, t\right)}{\longrightarrow} \mathbf{x}, \mathbf{n}_{r}=\mathbf{x}_{r}-\mathbf{x}
$$

The dependent variables in the reaction rate equations are the concentrations of the chemical compounds. The concentration of $X_{i}$ is denoted by $\left[x_{i}\right]$ and collected in $[\mathbf{x}] \in \mathbb{R}_{+}^{N}$, where $\mathbb{R}_{+}$denotes a non-negative real number. Then the reaction rate equations for $N$ species and $R$ reactions are [16]

$$
\frac{d\left[x_{i}\right]}{d t}=-\sum_{r=1}^{R} n_{r i} w_{r}([\mathbf{x}], t), i=1 \ldots N .
$$

The equations (2) are valid when the molecular copy number of each species is large.

The master equation is a differential-difference equation for the time evolution of the $\mathrm{PDF} p(\mathbf{x}, t)$ for the probability that the molecular state is $\mathbf{x}$ at time $t[16]$. Introduce a splitting of $\mathbf{n}_{r}$ into two parts as in [11] so that

$$
\mathbf{n}_{r}=\mathbf{n}_{r}^{+}+\mathbf{n}_{r}^{-}, n_{r i}^{+}=\max \left(n_{r i}, 0\right), n_{r i}^{-}=\min \left(n_{r i}, 0\right) .
$$

The inequality relation $\mathbf{x} \geq 0$ for a vector $\mathbf{x}$ denotes that all components are nonnegative. A reaction (1) at $\mathbf{x}_{r}$ takes place if $\mathbf{x}_{r} \geq 0$ and $\mathbf{x} \geq 0$ or equivalently $\mathbf{x}+\mathbf{n}_{r}^{-} \geq 0$, and a reaction at $\mathbf{x}$ occurs if $\mathbf{x} \geq 0$ and $\mathbf{x}-\mathbf{n}_{r} \geq 0$ or equivalently $\mathbf{x}-\mathbf{n}_{r}^{+} \geq 0$. Introduce the flux for the $r$ :th reaction

$$
q_{r}(\mathbf{x}, t)=w_{r}(\mathbf{x}, t) p(\mathbf{x}, t) .
$$

With $R$ reactions the master equation is

$$
\frac{\partial p(\mathbf{x}, t)}{\partial t}=\sum_{\substack{r=1 \\ \mathbf{x}+\mathbf{n}_{r}^{-} \geq 0}}^{R} q_{r}\left(\mathbf{x}+\mathbf{n}_{r}, t\right)-\sum_{\substack{r=1 \\ \mathbf{x}-\mathbf{n}_{r}^{+} \geq 0}}^{R} q_{r}(\mathbf{x}, t) .
$$

The total probability $\sum_{\mathbf{x} \geq 0} p(\mathbf{x}, t)$ is conserved by the master equation [11]. 
The FP equation (or forward Kolmogorov equation) is derived by first letting $\mathbf{x} \in \mathbb{R}_{+}^{N}$, then expanding the first sum of the master equation (3) in its Taylor series (the Kramers-Moyal expansion), and finally truncating after the second order term $[12,16,23]$. The FP equation for the PDF is

$$
\begin{aligned}
\frac{\partial p(\mathbf{x}, t)}{\partial t} & =\sum_{r=1}^{R}\left\{\sum_{i=1}^{N} n_{r i} \frac{\partial q_{r}(\mathbf{x}, t)}{\partial x_{i}}+\sum_{i=1}^{N} \sum_{j=1}^{N} \frac{n_{r i} n_{r j}}{2} \frac{\partial^{2} q_{r}(\mathbf{x}, t)}{\partial x_{i} \partial x_{j}}\right\} \\
& =\sum_{r=1}^{R}\left\{\sum_{i=1}^{N} n_{r i} \frac{\partial}{\partial x_{i}}\left(q_{r}(\mathbf{x}, t)+\frac{1}{2} \sum_{j=1}^{N} n_{r j} \frac{\partial q_{r}(\mathbf{x}, t)}{\partial x_{j}}\right)\right\} .
\end{aligned}
$$

The conservation form of the FP equation is obtained by introducing $\mathbf{F}_{r}$ with the components

$$
F_{r i}=n_{r i}\left(q_{r}+0.5 \mathbf{n}_{r} \cdot \nabla q_{r}\right), r=1 \ldots R, i=1 \ldots N .
$$

Then by (4)

$$
\frac{\partial p(\mathbf{x}, t)}{\partial t}=\sum_{r=1}^{R} \nabla \cdot \mathbf{F}_{r}
$$

The quantity $\sum_{r} \mathbf{F}_{r}$ is denoted the probability current in [12] and is the flux function when (5) is regarded as a conservation law. Let the boundaries of the domain be denoted by $\Gamma_{i}=\left\{\mathbf{x} \mid \mathbf{x} \in \mathbb{R}_{+}^{N}\right.$ and $\left.x_{i}=0\right\}$. With the boundary conditions (reflecting barrier conditions in [12])

$$
\sum_{r=1}^{R} n_{r i}\left(q_{r}+0.5 \mathbf{n}_{r} \cdot \nabla q_{r}\right)=0 \text { on } \Gamma_{i}, i=1 \ldots N
$$

and $\mathbf{F}_{r}=0$ far away from the origin, the total probability $\int_{\mathbf{x} \geq 0} p(\mathbf{x}, t) d \mathbf{x}$ is conserved for all $t$ by the FP equation [11]. An alternative is to apply absorbing barrier conditions [12]

$$
p(\mathbf{x}, t)=0 \text { on } \Gamma_{i}, i=1 \ldots N .
$$

Then the total probability is not necessarily preserved.

\section{Dimensional reduction of the Fokker-Planck equation}

By deriving equations for the expected values of certain species, the dimension of the problem is reduced and becomes tractable while retaining full spatial dependence for a few of the molecular species in the master or $\mathrm{FP}$ equations. 


\subsection{Simplifying assumptions}

In order to reduce the number of dimensions in $\mathbf{x}$, introduce a splitting such that $\mathbf{x}^{T} \rightarrow\left(\mathbf{x}^{T}, \mathbf{y}^{T}\right)$ and $\mathbf{n}_{r}^{T} \rightarrow\left(\mathbf{m}_{r}^{T}, \mathbf{n}_{r}^{T}\right)$, where $\mathbf{x} \in \mathbb{R}_{+}^{m}, \mathbf{y} \in \mathbb{R}^{n}, \mathbf{m}_{r} \in \mathbb{Z}^{m}, \mathbf{n}_{r} \in \mathbb{Z}^{n}$, and $N=m+n$. Assume that the two sets of stochastic variables $X_{i}, i=1 \ldots m$, and $Y_{j}, j=1 \ldots n$, are independent such that the corresponding PDF is

$$
p(\mathbf{x}, \mathbf{y}, t)=p_{X}(\mathbf{x}, t) p_{Y}(\mathbf{y}, t) .
$$

The PDF $p$ is zero for $\mathbf{x}$ far away from the origin, i.e.

$$
p(\mathbf{x}, \mathbf{y}, t)=0 \text { for } S_{\eta}=\left\{\mathbf{x} \mid \mathbf{x} \in \mathbb{R}_{+}^{m},\|\mathbf{x}\|_{2}>\eta\right\},
$$

for some $\eta>0$. Furthermore, assume that the $Y_{j}$-variables are normally distributed and independent resulting in

$$
p(\mathbf{x}, \mathbf{y}, t)=\gamma_{n} p_{X}(\mathbf{x}, t) \exp \left(-\sum_{j=1}^{n} \frac{\left(y_{j}-\phi_{j}\right)^{2}}{2 \sigma^{2}}\right) .
$$

The assumptions on $\boldsymbol{\phi}$ is that it is bounded in $\mathbf{x}$ and $t$ and has bounded first and second derivatives in $\mathbf{x}$ so that $\phi$ belongs to the Sobolev space [22]

$$
\phi \in W^{2, \infty}\left(Q_{\eta}\right), Q_{\eta}=\mathbb{R}_{+}^{m} \backslash S_{\eta} .
$$

The parameter $\sigma$ is small and constant in the approximation. If the number of molecules of a certain kind is large, then the corresponding variance is small [16].

The marginal PDF $p_{0}$ is defined by

$$
p_{0}(\mathbf{x}, t)=\int p(\mathbf{x}, \mathbf{y}, t) d \mathbf{y}=\gamma_{n} p_{X}(\mathbf{x}, t) \int \exp \left(-\sum_{j=1}^{n} \frac{\left(y_{j}-\phi_{j}\right)^{2}}{2 \sigma^{2}}\right) d \mathbf{y}
$$

The integration $\int$ as in $(11)$ is taken over $\mathbb{R}^{n}$. The normalization constant $\gamma_{n}$ satisfies

$$
\gamma_{n}=\left(2 \sigma^{2} \pi\right)^{-n / 2}
$$

for $\mathbf{y} \in \mathbb{R}^{n}$ so that

$$
\gamma_{n} \int \exp \left(-\sum_{j=1}^{n} \frac{\left(y_{j}-\phi_{j}\right)^{2}}{2 \sigma^{2}}\right) d \mathbf{y}=1
$$

and $p_{0}(\mathbf{x}, t)=p_{X}(\mathbf{x}, t)$. Since $p$ and $p_{0}$ are non-negative and

$$
\iint_{\mathbb{R}_{+}^{m}} p(\mathbf{x}, \mathbf{y}, t) d \mathbf{x} d \mathbf{y}=\int_{\mathbb{R}_{+}^{m}} p_{0}(\mathbf{x}, t) d \mathbf{x}=1
$$


both $p$ and $p_{0}$ are PDFs. The first partial moment for $y_{k}$ with respect to $\mathbf{y}$ is

$$
\begin{aligned}
p_{k}(\mathbf{x}, t) & =\int y_{k} p(\mathbf{x}, \mathbf{y}, t) d \mathbf{y}=\gamma_{n} p_{0}(\mathbf{x}, t) \int y_{k} \exp \left(-\sum_{j=1}^{n} \frac{\left(y_{k}-\phi_{k}\right)^{2}}{2 \sigma^{2}}\right) d \mathbf{y} \\
& =p_{0}(\mathbf{x}, t) \gamma_{1} \int y_{k} \exp \left(-\frac{\left(y_{k}-\phi_{k}\right)^{2}}{2 \sigma^{2}}\right) d y_{k} \\
& =p_{0}(\mathbf{x}, t) \gamma_{1} \int\left(z_{k} \sigma \sqrt{2}+\phi_{k}\right) \exp \left(-z_{k}^{2}\right) \sigma \sqrt{2} d z_{k} \\
& =p_{0}(\mathbf{x}, t) \phi_{k}(\mathbf{x}, t) \gamma_{1} \int \exp \left(-z_{k}^{2}\right) \sigma \sqrt{2} d z_{k}=p_{0}(\mathbf{x}, t) \phi_{k}(\mathbf{x}, t) .
\end{aligned}
$$

The conditional expected value of $y_{k}$ is $\phi_{k}$ since

$$
E\left[y_{k} \mid \mathbf{x}\right]=\int y_{k} p(\mathbf{x}, \mathbf{y}, t) d \mathbf{y} / \int p(\mathbf{x}, \mathbf{y}, t) d \mathbf{y}=p_{k}(\mathbf{x}, t) / p_{0}(\mathbf{x}, t)=\phi_{k}(\mathbf{x}, t) .
$$

The following lemma relates $E[w \mid \mathbf{x}]$ to $w$ and is needed to obtain approximate differential-difference equations for $p_{0}$ and the moments $p_{k}$.

Lemma 3.1. Assume that

$$
w(\mathbf{x}, \boldsymbol{\phi}+\Delta \boldsymbol{\phi}, t)=w(\mathbf{x}, \boldsymbol{\phi}, t)+\sum_{j=1}^{n} c_{j}(\mathbf{x}, \boldsymbol{\phi}, t) \Delta \phi_{j}+\rho(\mathbf{x}, \boldsymbol{\phi}, \Delta \boldsymbol{\phi}, t)
$$

for $\phi \in \mathbb{R}^{n}$, and let the conditional expected value of $w$ be

$$
E[w \mid \mathbf{x}]=\gamma_{n} \int w(\mathbf{x}, \mathbf{y}, t) \exp \left(-\sum_{j=1}^{n} \frac{\left(y_{j}-\phi_{j}\right)^{2}}{2 \sigma^{2}}\right) d \mathbf{y} .
$$

If $\delta_{j} \in \mathbb{Z}_{+},|\boldsymbol{\delta}|=\sum_{j} \delta_{j}$, and

$$
|\rho(\mathbf{x}, \boldsymbol{\phi}, \Delta \boldsymbol{\phi}, t)| \leq \sum_{k=2}^{q} \sum_{|\boldsymbol{\delta}|=k} \xi_{\boldsymbol{\delta}}(\mathbf{x}, t) \prod_{j=1}^{n}\left|\Delta \phi_{j}\right|^{\delta_{j}},
$$

then

$$
|E[w \mid \mathbf{x}]-w(\mathbf{x}, \boldsymbol{\phi}, t)| \leq \kappa_{1} \sigma^{2}\left(1-\sigma^{q-1}\right) /(1-\sigma),
$$

where $\kappa_{1}$ depends on $\mathbf{x}, t$, and $n$ and is independent of $\sigma$.

The proof of the lemma is found in the appendix.

The propensities $w_{r}$ in the chemical reactions (1) are usually polynomials or rational polynomials in $\mathbf{x}$ and $\mathbf{y}$ without poles for $\mathbf{x} \in \mathbb{R}_{+}^{m}$ and $\mathbf{y} \in \mathbb{R}^{n}$ with time dependent coefficients. They possess a bound on the remainder term $\rho$ as in the lemma with a limited $q$. If they are independent of or linear in $\mathbf{y}$, then $E[w \mid \mathbf{x}]=w(\mathbf{x}, \boldsymbol{\phi}, t)$. If $\sigma<1$, then the difference between $E[w \mid \mathbf{x}]$ and $w(\mathbf{x}, \boldsymbol{\phi}, t)$ is proportional to $\sigma^{2}$. 


\subsection{Differential equations}

After defining $p_{0}$ in (11) and $p_{k}$ in (13) we derive differential-difference equations and the corresponding FP equations satisfied by them. The equation for $p_{0}$ is obtained for one reaction $r$ from the master equation (3)

$$
\begin{aligned}
& \frac{\partial p_{0}(\mathbf{x}, t)}{\partial t}=\int \frac{\partial p}{\partial t} d \mathbf{y}=\int q_{r}\left(\mathbf{x}+\mathbf{m}_{r}, \mathbf{y}+\mathbf{n}_{r}, t\right)-q_{r}(\mathbf{x}, \mathbf{y}, t) d \mathbf{y} \\
& =\int w_{r}\left(\mathbf{x}+\mathbf{m}_{r}, \mathbf{y}+\mathbf{n}_{r}, t\right) \gamma_{n} p_{0}\left(\mathbf{x}+\mathbf{m}_{r}, t\right) \exp \left(-\sum_{j=1}^{n} \frac{\left(y_{j}+n_{r j}-\phi_{j}\right)^{2}}{2 \sigma^{2}}\right) \\
& -w_{r}(\mathbf{x}, \mathbf{y}, t) \gamma_{n} p_{0}(\mathbf{x}, t) \exp \left(-\sum_{j=1}^{n} \frac{\left(y_{j}-\phi_{j}\right)^{2}}{2 \sigma^{2}}\right) d \mathbf{y} \\
& =w_{r}\left(\mathbf{x}+\mathbf{m}_{r}, \boldsymbol{\phi}\left(\mathbf{x}+\mathbf{m}_{r}, t\right), t\right) p_{0}\left(\mathbf{x}+\mathbf{m}_{r}, t\right)-w_{r}(\mathbf{x}, \boldsymbol{\phi}(\mathbf{x}, t), t) p_{0}(\mathbf{x}, t)+\rho_{0}\left(p_{0}\right) .
\end{aligned}
$$

The functional $\rho_{0}$ is bounded by Lemma 3.1 and vanishes like $\sigma^{2}$ for a bounded $p_{0}$ and a small $\sigma$.

The corresponding FP equation is

$$
\frac{\partial p_{0}(\mathbf{x}, t)}{\partial t}=\left\{\sum_{i=1}^{m} m_{r i} \frac{\partial q_{r 0}(\mathbf{x}, t)}{\partial x_{i}}+\sum_{i=1}^{m} \sum_{j=1}^{m} \frac{m_{r i} m_{r j}}{2} \frac{\partial^{2} q_{r 0}(\mathbf{x}, t)}{\partial x_{i} \partial x_{j}}\right\}+\rho_{0}\left(p_{0}\right)
$$

with $q_{r 0}(\mathbf{x}, t)=w_{r}(\mathbf{x}, \boldsymbol{\phi}(\mathbf{x}, t), t) p_{0}(\mathbf{x}, t)$, cf. (4). Let $\mathbf{F}_{r 0}$ consist of the elements

$$
F_{r 0 i}=m_{r i}\left(q_{r 0}+0.5 \mathbf{m}_{r} \cdot \nabla q_{r 0}\right), r=1 \ldots R, i=1 \ldots m .
$$

Then the FP equation for $p_{0}$ with $R$ reactions is

$$
\frac{\partial p_{0}(\mathbf{x}, t)}{\partial t}=\sum_{r=1}^{R} \nabla \cdot \mathbf{F}_{r 0}
$$

where $\rho_{0}$ is ignored, cf. (5). Since $p_{0}$ is a PDF, its total mass should be conserved over $\mathbb{R}_{+}^{m}$. It follows from the assumption (8) on $p$ and (11) that $p_{0}(\mathbf{x}, t)=0$ when $\mathbf{x} \in S_{\eta}$ and therefore $\nabla p_{0}=0$. By the definition of $\mathbf{F}_{r 0}$ we have $\mathbf{F}_{r 0}=0$ in $S_{\eta}$. The time evolution of the total mass of $p_{0}$ is obtained by Gauss' formula

$$
\frac{\partial}{\partial t} \int_{\mathbb{R}_{+}^{m}} p_{0}(\mathbf{x}, t) d \mathbf{x}=\int_{\mathbb{R}_{+}^{m}} \nabla \cdot \sum_{r=1}^{R} \mathbf{F}_{r 0} d \mathbf{x}=\int_{\Gamma_{i}} \sum_{r=1}^{R} \hat{\mathbf{n}}_{i} \cdot \mathbf{F}_{r 0} d \mathbf{x}=0,
$$

where $\hat{\mathbf{n}}_{i}$ is the zero vector except for the $i$ :th element which is -1 . This is the normal direction of $\Gamma_{i}$. Then the conclusion from (18) is that suitable boundary conditions for (17) to preserve the total probability are

$$
\sum_{r=1}^{R} m_{r i}\left(q_{r 0}+0.5 \mathbf{m}_{r} \cdot \nabla q_{r 0}\right)=0 \text { on } \Gamma_{i}, i=1 \ldots m,
$$


(cf. (6)).

The differential-difference equation satisfied by the first partial moments is derived for a single reaction $r$ from the master equation (3) in the same manner as in (15)

$$
\begin{aligned}
\frac{\partial p_{k}(\mathbf{x}, t)}{\partial t}= & \int y_{k} \frac{\partial p}{\partial t} d \mathbf{y}=\int y_{k} q_{r}\left(\mathbf{x}+\mathbf{m}_{r}, \mathbf{y}+\mathbf{n}_{r}, t\right)-y_{k} q_{r}(\mathbf{x}, \mathbf{y}, t) d \mathbf{y} \\
= & \int\left(y_{k}-n_{r k}\right) q_{r}\left(\mathbf{x}+\mathbf{m}_{r}, \mathbf{y}, t\right)-y_{k} q_{r}(\mathbf{x}, \mathbf{y}, t) d \mathbf{y} \\
= & \phi_{k}\left(\mathbf{x}+\mathbf{m}_{r}, t\right) w_{r}\left(\mathbf{x}+\mathbf{m}_{r}, \boldsymbol{\phi}\left(\mathbf{x}+\mathbf{m}_{r}, t\right), t\right) p_{0}\left(\mathbf{x}+\mathbf{m}_{r}, t\right) \\
& -\phi_{k}(\mathbf{x}, t) w_{r}(\mathbf{x}, \boldsymbol{\phi}(\mathbf{x}, t), t) p_{0}(\mathbf{x}, t) \\
& -n_{r k} w_{r}\left(\mathbf{x}+\mathbf{m}_{r}, \boldsymbol{\phi}\left(\mathbf{x}+\mathbf{m}_{r}, t\right), t\right) p_{0}\left(\mathbf{x}+\mathbf{m}_{r}, t\right)+\rho_{k}\left(p_{0}, p_{k}\right) \\
= & w_{r}\left(\mathbf{x}+\mathbf{m}_{r}, \boldsymbol{\phi}\left(\mathbf{x}+\mathbf{m}_{r}, t\right), t\right) p_{k}\left(\mathbf{x}+\mathbf{m}_{r}, t\right)-w_{r}(\mathbf{x}, \boldsymbol{\phi}(\mathbf{x}, t), t) p_{k}(\mathbf{x}, t) \\
& -n_{r k} w_{r}\left(\mathbf{x}+\mathbf{m}_{r}, \boldsymbol{\phi}\left(\mathbf{x}+\mathbf{m}_{r}, t\right), t\right) p_{0}\left(\mathbf{x}+\mathbf{m}_{r}, t\right)+\rho_{k}\left(p_{0}, p_{k}\right) .
\end{aligned}
$$

As in (15), for bounded $p_{0}$ and $p_{k}, \rho_{k}$ is bounded by Lemma 3.1 and is of $\mathcal{O}\left(\sigma^{2}\right)$ for a small $\sigma$. The FP approximation of (20) is

$$
\begin{aligned}
\frac{\partial p_{k}(\mathbf{x}, t)}{\partial t}= & \sum_{i=1}^{m} m_{r i} \frac{\partial q_{r k}(\mathbf{x}, t)}{\partial x_{i}}+\sum_{i=1}^{m} \sum_{j=1}^{m} \frac{m_{r i} m_{r j}}{2} \frac{\partial^{2} q_{r k}(\mathbf{x}, t)}{\partial x_{i} \partial x_{j}} \\
& -n_{r k} w_{r}\left(\mathbf{x}+\mathbf{m}_{r}, \boldsymbol{\phi}\left(\mathbf{x}+\mathbf{m}_{r}, t\right), t\right) p_{0}\left(\mathbf{x}+\mathbf{m}_{r}, t\right)+\rho_{k},
\end{aligned}
$$

with $q_{r k}(\mathbf{x}, t)=w_{r}(\mathbf{x}, \boldsymbol{\phi}(\mathbf{x}, t), t) p_{k}(\mathbf{x}, t)$. With $R$ reactions and the components

$$
F_{r k i}=m_{r i}\left(q_{r k}+0.5 \mathbf{m}_{r} \cdot \nabla q_{r k}\right), i=1 \ldots m, k=1 \ldots n, r=1 \ldots R,
$$

in $\mathbf{F}_{r k}$, the $\mathrm{FP}$ equation for $p_{k}$ with $R$ reactions ignoring $\rho_{k}$ is

$$
\frac{\partial p_{k}(\mathbf{x}, t)}{\partial t}=\sum_{r=1}^{R} \nabla \cdot \mathbf{F}_{r k}-\sum_{r=1}^{R} n_{r k} w_{r}\left(\mathbf{x}+\mathbf{m}_{r}, \boldsymbol{\phi}\left(\mathbf{x}+\mathbf{m}_{r}, t\right), t\right) p_{0}\left(\mathbf{x}+\mathbf{m}_{r}, t\right) .
$$

The following theorem provides bounds on the differences between the solutions to (16) and (17) and the solutions to (21) and (22) due to the variance parameter $\sigma$ in (9). The solutions are weak solutions of the PDEs [1, 22].

Theorem 3.2. Assume that $w_{r}, r=1 \ldots R$, is bounded and has bounded first and second derivatives in $\mathbf{x}$ and $\mathbf{y}$ in $Q_{\eta} \times[0, T]$, that $\boldsymbol{\phi}$ satisfies (10) and that $M(\mathbf{x}, t)=\sum_{r} w_{r} \mathbf{m}_{r} \mathbf{m}_{r}^{T} \in \mathbb{R}^{m \times m}$ is positive definite.

Let $\tilde{p}_{0}$ be the solution of (16) and $p_{0}$ the solution of (17) with the boundary conditions $\tilde{p}_{0}(\mathbf{x}, t)=0$ and $p_{0}(\mathbf{x}, t)=0$ on the boundary $\partial Q_{\eta}$ of $Q_{\eta}$ and identical initial conditions at $t=0$. Then for $\sigma$ sufficiently small and $t \in[0, T]$

$$
\left|\tilde{p}_{0}(\mathbf{x}, t)-p_{0}(\mathbf{x}, t)\right| \leq C_{0} \sigma^{2},
$$


where $\sigma$ is the variance in (11) and $C_{0}$ depends on $\eta, T, w_{r}, \boldsymbol{\phi}, m$, and $\mathbf{m}_{r}$.

Let $\tilde{p}_{k}$ be the solution of (21) and $p_{k}$ the solution of (22) with the boundary conditions $\tilde{p}_{k}(\mathbf{x}, t)=0$ and $p_{k}(\mathbf{x}, t)=0$ on the boundary $\partial Q_{\eta}$ and the same initial conditions at $t=0$. Then for $\sigma$ sufficiently small and $t \in[0, T]$

$$
\left|\tilde{p}_{k}(\mathbf{x}, t)-p_{k}(\mathbf{x}, t)\right| \leq C_{k} \sigma^{2},
$$

where $C_{k}$ depends on $\eta, T, w_{r}, \boldsymbol{\phi}, m$, and $\mathbf{m}_{r}$.

Proof. Let $w_{r}=w_{r}(\mathbf{x}, \boldsymbol{\phi}(\mathbf{x}, t), t)$ and

$$
\begin{aligned}
\mathcal{A}(p, \nabla p)= & 0.5 \sum_{r} w_{r} \mathbf{m}_{r} \mathbf{m}_{r}^{T} \nabla p=0.5 M \nabla p, \\
\mathcal{B}_{0}(p, \nabla p)= & \left(\sum_{r}\left(\mathbf{m}_{r} \cdot \nabla\right)\left(w_{r}+0.5\left(\mathbf{m}_{r} \cdot \nabla\right) w_{r}\right)\right) p \\
& +\left(\sum_{r}\left(w_{r}+0.5\left(\mathbf{m}_{r} \cdot \nabla\right) w_{r}\right) \mathbf{m}_{r}\right) \cdot \nabla p .
\end{aligned}
$$

Then the equation for $p_{0}$ in (17) is

$$
\frac{\partial p_{0}}{\partial t}=\nabla \cdot \mathcal{A}\left(p_{0}, \nabla p_{0}\right)+\mathcal{B}_{0}\left(p_{0}, \nabla p_{0}\right)
$$

Since $M$ is positive definite, there is an $a$ such that $\mathbf{u}^{T} \mathcal{A}(p, \mathbf{u}) \geq a \mathbf{u}^{T} \mathbf{u}$. Moreover, there are $\bar{a}, c(\mathbf{x}, t)$, and $d(\mathbf{x}, t)$ in the bounds

$$
|\mathcal{A}(p, \mathbf{u})| \leq \bar{a}\|\mathbf{u}\|_{2},\left|\mathcal{B}_{0}(p, \mathbf{u})\right| \leq c\|\mathbf{u}\|_{2}+d|p|
$$

From the bounds on $w_{r}$ and $\phi$ and their derivatives, there is a constant $\bar{a}$ and $c(\mathbf{x}, t)$ and $d(\mathbf{x}, t)$ are bounded in the norm

$$
\|f\|_{\infty}=\sup _{t \in[0, T]} \sup _{\mathbf{x} \in Q_{\eta}}|f(\mathbf{x}, t)| .
$$

The maximum principle in Theorem 1 in [1] provides an upper bound on $p_{0}$. A lower bound is obtained by applying the maximum principle to the equation for $-p_{0}$. Therefore, $\left\|p_{0}\right\|_{\infty}$ is bounded.

The equation for $p_{k}$ in (22) has the same spatial differential operator as $p_{0}$ in (23) plus a source term so that

$$
\mathcal{B}_{k}\left(p_{k}, \nabla p_{k}\right)=\mathcal{B}_{0}\left(p_{k}, \nabla p_{k}\right)-\sum_{r=1}^{R} n_{r k} w_{r}\left(\mathbf{x}+\mathbf{m}_{r}, \phi\left(\mathbf{x}+\mathbf{m}_{r}, t\right), t\right) p_{0} .
$$

There is a bound on $\left|\mathcal{B}_{k}\right|$ as in (24) with a term $g_{1}(\mathbf{x}, t)$ bounding the sum in (25). Since $\left\|w_{r}\right\|_{\infty}$ and $\left\|p_{0}\right\|_{\infty}$ are bounded, so is $\left\|g_{1}\right\|_{\infty}$. Consequently, according to the maximum principle in [1] there is also a bound on $\left\|p_{k}\right\|_{\infty}$.

The difference $\delta p_{0}=\tilde{p_{0}}-p_{0}$ satisfies (23) with $\delta p_{0}=0$ initially and on the boundary and $g_{2}=\rho_{0}\left(p_{0}\right)$ in the right hand side. By Taylor's formula and 
the bounds on $w_{r}$ and $\boldsymbol{\phi}$, the assumptions on $w$ in Lemma 3.1 are fulfilled and $\left\|g_{2}\right\|_{\infty} \leq C_{g} \sigma^{2}\left\|p_{0}\right\|_{\infty}$. Hence, by the maximum principle we have

$$
\delta p_{0} \leq C_{0}^{\prime}\left\|g_{2}\right\|_{\infty} \leq C_{0} \sigma^{2}
$$

The same bound for $-\delta p_{0}$ is obtained by applying the maximum principle to the equation for $-\delta p_{0}$.

The bound on $\left|\delta p_{k}\right|$ follows from the maximum principle in [1] and Lemma 3.1 in the same manner.

The sufficient conditions in the theorem are often satisfied by chemical reactions. To be able to apply the maximum principle separately to the equations for $p_{0}$ and $p_{k}$, the coupling $\phi$ between the equations is assumed to be smooth. The difference between the solutions with and without the remainder term $\rho$ can be made as small as we wish by choosing a small $\sigma$.

\subsection{Back to the reaction rate equations}

The equations can be simplified further by considering the expected value of $\phi_{j}$ or equivalently the expected value of $y_{k}$ defined by

$$
\bar{\phi}_{k}(t)=\int_{\mathbb{R}_{+}^{m}} \phi_{k}(\mathbf{x}, t) p_{0}(\mathbf{x}, t) d \mathbf{x}=\int_{\mathbb{R}_{+}^{m}} p_{k}(\mathbf{x}, t) d \mathbf{x}=\iint_{\mathbb{R}_{+}^{m}} y_{k} p(\mathbf{x}, \mathbf{y}, t) d \mathbf{x} d \mathbf{y}
$$

Integrate (22) over $\mathbb{R}_{+}^{m}$ to obtain

$$
\begin{aligned}
\frac{d \bar{\phi}_{k}}{d t}= & \int_{\mathbb{R}_{+}^{m}} \frac{\partial p_{k}}{\partial t} d \mathbf{x}=\sum_{r=1}^{R} \int_{\mathbb{R}_{+}^{m}} \nabla \cdot \mathbf{F}_{r k} d \mathbf{x} \\
& -\sum_{r=1}^{R} \int_{\mathbb{R}_{+}^{m}} n_{r k} w_{r}\left(\mathbf{x}+\mathbf{m}_{r}, \boldsymbol{\phi}\left(\mathbf{x}+\mathbf{m}_{r}, t\right), t\right) p_{0}\left(\mathbf{x}+\mathbf{m}_{r}, t\right) d \mathbf{x} .
\end{aligned}
$$

Let the boundary conditions for $p_{k}$ be

$$
\sum_{r=1}^{R} m_{r i}\left(q_{r k}+0.5 \mathbf{m}_{r} \cdot \nabla q_{r k}\right)=0 \text { on } \Gamma_{i}, i=1 \ldots m
$$

and since $p_{0}(\mathbf{x}, t)=0$ for $\mathbf{x} \in S_{\eta}$ we also have $p_{k}(\mathbf{x}, t)=0$ and $\partial p_{k} / \partial x_{i}=0$ for $\mathbf{x} \in S_{\eta}$. After integration with Gauss' formula, the system of differential 
equations for $\bar{\phi}$ is from $(27)$

$$
\begin{aligned}
\frac{d \bar{\phi}_{k}}{d t}= & \sum_{i=1}^{m} \int_{\Gamma_{i}} \sum_{r=1}^{R} \hat{\mathbf{n}}_{i} \cdot \mathbf{F}_{r k} d \mathbf{x} \\
& -\sum_{r=1}^{R} n_{r k} \int_{\mathbb{R}_{+}^{m}} w_{r}\left(\mathbf{x}+\mathbf{m}_{r}, \boldsymbol{\phi}\left(\mathbf{x}+\mathbf{m}_{r}, t\right), t\right) p_{0}\left(\mathbf{x}+\mathbf{m}_{r}, t\right) d \mathbf{x} \\
= & -\sum_{r=1}^{R} n_{r k} \int_{\mathbb{R}_{+}^{m}} w_{r}\left(\mathbf{x}+\mathbf{m}_{r}, \boldsymbol{\phi}\left(\mathbf{x}+\mathbf{m}_{r}, t\right), t\right) p_{0}\left(\mathbf{x}+\mathbf{m}_{r}, t\right) d \mathbf{x}, \\
& k=1 \ldots n .
\end{aligned}
$$

The last integral in (29) is the expected value of $w_{r}$ after a shift with $\mathbf{m}_{r}$.

In order to close the system, assume that $p_{0}$ has a special form

$$
p_{0}(\mathbf{x}, t)=\gamma_{m} \exp \left(-\sum_{j=1}^{m} \frac{\left(x_{j}-\psi_{j}\right)^{2}}{2 \sigma^{2}}\right)
$$

with time dependent positive values $\psi_{j}, j=1 \ldots m$, and a small $\sigma$ implying that $p_{0}(\mathbf{x}, t)$ is small for $\mathbf{x}$ close to $\Gamma_{i}$. Then

$$
\begin{aligned}
& \int_{\mathbb{R}_{+}^{m}} w_{r}\left(\mathbf{x}+\mathbf{m}_{r}, \boldsymbol{\phi}\left(\mathbf{x}+\mathbf{m}_{r}, t\right), t\right) p_{0}\left(\mathbf{x}+\mathbf{m}_{r}, t\right) d \mathbf{x} \\
& =\gamma_{m} \int_{\mathbb{R}^{m}} w_{r}\left(\mathbf{x}+\mathbf{m}_{r}, \boldsymbol{\phi}\left(\mathbf{x}+\mathbf{m}_{r}, t\right), t\right) \exp \left(-\sum_{j=1}^{m} \frac{\left(x_{j}+m_{r j}-\psi_{j}\right)^{2}}{2 \sigma^{2}}\right) d \mathbf{x}+\rho_{w b d} \\
& =w_{r}(\boldsymbol{\psi}(t), \boldsymbol{\phi}(\boldsymbol{\psi}(t), t), t)+\rho_{w}+\rho_{w b d} .
\end{aligned}
$$

Here, $\rho_{w}$ is small for a small $\sigma$ by Lemma 3.1 and the correction $\rho_{w b d}$ due to the removal of the boundary is also small when $\sigma$ is small and is bounded in the next lemma.

Lemma 3.3. Assume that

$$
|w(\mathbf{x}+\Delta \mathbf{x}, t)| \leq \sum_{k=0}^{q} \sum_{|\boldsymbol{\delta}|=k} \eta_{\boldsymbol{\delta}}(\mathbf{x}, t) \prod_{j=1}^{m}\left|\Delta x_{j}\right|^{\delta_{j}}
$$

for $\Delta x_{j} \in \mathbb{R}, \delta_{j} \in \mathbb{Z}_{+}, j=1 \ldots m$, and $|\boldsymbol{\delta}|=\sum_{j} \delta_{j}$. Let the expected value of $w$ be

$$
E[w]=\gamma_{m} \int_{\mathbb{R}_{+}^{m}} w(\mathbf{x}+\mathbf{m}, t) \exp \left(-\sum_{j=1}^{m} \frac{\left(x_{j}+m_{r j}-\psi_{j}\right)^{2}}{2 \sigma^{2}}\right) d \mathbf{x} .
$$

Furthermore, assume that $m_{r j}-\psi_{j}<0$ for all $j$ and let

$$
I_{w}=\gamma_{m} \int_{\mathbb{R}^{m}} w(\mathbf{x}+\mathbf{m}, t) \exp \left(-\sum_{j=1}^{m} \frac{\left(x_{j}+m_{r j}-\psi_{j}\right)^{2}}{2 \sigma^{2}}\right) d \mathbf{x} .
$$


Then

$$
\left|E[w]-I_{w}\right| \leq \kappa_{3} \exp \left(-\frac{\min _{j}\left(\psi_{j}-m_{r j}\right)^{2}}{2 \sigma^{2}}\right),
$$

where $\kappa_{3}$ is polynomial in $\left(\psi_{j}-m_{r j}\right) / \sigma$ of degree at most $q-1$ with coefficients depending on $\mathbf{x}, t, q$, and $m$.

The proof of the lemma is found in the appendix.

As in Lemma 3.1, the condition on $w$ is usually satisfied by the propensities in models for chemical reactions. The difference between integration over the positive orthant for $E[w]$ and the whole space for $I_{w}$ decreases rapidly for decreasing $\sigma$.

With the assumption (10) on $\boldsymbol{\phi}$, it follows from (26), (30) with $w_{r}=\phi_{k}(\mathbf{x}, t)$, Lemma 3.1 and Lemma 3.3 that for small $\sigma$

$$
\bar{\phi}_{k}(t)=\phi_{k}(\boldsymbol{\psi}(t), t)+\rho_{\phi}+\rho_{\phi b d} \approx \phi_{k}(\boldsymbol{\psi}(t), t) .
$$

Insert (31) into (30), sum over all reactions as in (29), and ignore terms that vanish with vanishing $\sigma$ to arrive at

$$
\frac{d \bar{\phi}_{k}}{d t}=-\sum_{r=1}^{R} n_{r k} w_{r}(\boldsymbol{\psi}(t), \bar{\phi}(t), t), k=1 \ldots n .
$$

The first moment of $x_{k}$ over $\mathbb{R}^{m}$ is

$$
\int_{\mathbb{R}^{m}} x_{k} p_{0}(\mathbf{x}, t) d \mathbf{x}=\gamma_{m} \int_{\mathbb{R}^{m}} x_{k} \exp \left(-\sum_{j=1}^{m} \frac{\left(x_{j}-\psi_{j}\right)^{2}}{2 \sigma^{2}}\right) d \mathbf{x}=\psi_{k}, k=1 \ldots m .
$$

By Lemma 3.3 with $w=x_{k}$, the difference between the expected value of $x_{k}$ defined by

$$
E\left[x_{k}\right]=\int_{\mathbb{R}_{+}^{m}} x_{k} p_{0}(\mathbf{x}, t) d \mathbf{x}
$$

and $\psi_{k}$ is

$$
\left|E\left[x_{k}\right]-\psi_{k}\right|=\left|\int_{\mathbb{R}^{m} \backslash \mathbb{R}_{+}^{m}} x_{k} p_{0}(\mathbf{x}, t) d \mathbf{x}\right| \leq \kappa_{3} \exp \left(-0.5 \min _{j}\left(\psi_{j}-m_{r j}\right)^{2} / \sigma^{2}\right) .
$$

The difference vanishes exponentially fast when $\sigma \rightarrow 0$.

The differential-difference equation satisfied by $\boldsymbol{\psi}(t)$ for a single reaction $r$ is

$$
\begin{aligned}
\frac{d \psi_{k}}{d t} & =\int_{\mathbb{R}^{m}} x_{k} \frac{\partial p_{0}}{\partial t} d \mathbf{x}=\int_{\mathbb{R}^{m}} x_{k} q_{r 0}\left(\mathbf{x}+\mathbf{m}_{r}, t\right)-x_{k} q_{r 0}(\mathbf{x}, t) d \mathbf{x} \\
& =\int_{\mathbb{R}^{m}}\left(x_{k}-m_{r k}\right) q_{r 0}(\mathbf{x}, t) d \mathbf{x}-\int x_{k} q_{r 0}(\mathbf{x}, t) d \mathbf{x} \\
& =-\gamma_{m} m_{r k} \int_{\mathbb{R}^{m}} w_{r}(\mathbf{x}, \boldsymbol{\phi}(\mathbf{x}, t), t) \exp \left(-\sum_{j=1}^{m} \frac{\left(x_{j}-\psi_{j}\right)^{2}}{2 \sigma^{2}}\right) d \mathbf{x} \\
& =-m_{r k} w_{r}(\boldsymbol{\psi}, \boldsymbol{\phi}(\boldsymbol{\psi}, t), t)+\rho_{r \psi} .
\end{aligned}
$$


The remainder term $\rho_{r \psi}$ is bounded by Lemma 3.1. Replace $\phi$ by $\bar{\phi}$ in (34). The difference in the right hand side is bounded by a function in $\mathbf{x}$ and $t$ depending on $w_{r}$ times the sum of $\rho_{\phi}$ and $\rho_{\phi b d}$ in (31). Add the contributions from all $R$ reactions and ignore terms that vanish when $\sigma$ vanishes to arrive at

$$
\frac{d \psi_{k}(t)}{d t}=-\sum_{r=1}^{R} m_{r k} w_{r}(\boldsymbol{\psi}, \overline{\boldsymbol{\phi}}(t), t), k=1 \ldots m .
$$

The first moments of $\mathbf{x}$ in $\boldsymbol{\psi}$ satisfy a system of ODEs (35) and the first moments of $\mathbf{y}$ in $\overline{\boldsymbol{\phi}}$ in (26) also satisfy a system of ODEs (32). With $\left(\boldsymbol{\psi}^{T}, \overline{\boldsymbol{\phi}}^{T}\right) \rightarrow$ $[\mathbf{x}]^{T}$ and $\left(\mathbf{m}_{r}^{T}, \mathbf{n}_{r}^{T}\right) \rightarrow \mathbf{n}_{r}^{T}$ in (35) and (32) we have derived the macroscopic reaction rate equations $(2)$. The same equations are obtained directly with the assumption

$$
p(\mathbf{x}, \mathbf{y}, t)=\gamma_{m} \gamma_{n} \exp \left(-\sum_{j=1}^{m} \frac{\left(x_{j}-\psi_{j}\right)^{2}}{2 \sigma^{2}}\right) \exp \left(-\sum_{j=1}^{n} \frac{\left(y_{j}-\phi_{j}\right)^{2}}{2 \sigma^{2}}\right) .
$$

inserted into the master equation.

\subsection{Another simplification}

Another way of closing the system (29) is to assume that $\phi(\mathbf{x}, t)$ varies slowly with $\mathrm{x}$ so that $\Delta \boldsymbol{\phi}=\boldsymbol{\phi}(\mathbf{x}, t)-\bar{\phi}(t)$ is small. Then it follows with the assumption on $w_{r}$ in Lemma 3.1 that since $p_{0}$ is a PDF

$$
\begin{aligned}
& \mid \int_{\mathbb{R}_{+}^{m}} w_{r}\left(\mathbf{x}+\mathbf{m}_{r}, \boldsymbol{\phi}\left(\mathbf{x}+\mathbf{m}_{r}, t\right), t\right) p_{0}\left(\mathbf{x}+\mathbf{m}_{r}, t\right) d \mathbf{x} \\
& -\int_{\mathbb{R}_{+}^{m}} w_{r}\left(\mathbf{x}+\mathbf{m}_{r}, \bar{\phi}(t), t\right) p_{0}\left(\mathbf{x}+\mathbf{m}_{r}, t\right) d \mathbf{x} \mid \\
& \leq \int_{\mathbb{R}_{+}^{m}}\left|w_{r}\left(\mathbf{x}+\mathbf{m}_{r}, \boldsymbol{\phi}\left(\mathbf{x}+\mathbf{m}_{r}, t\right), t\right)-w_{r}\left(\mathbf{x}+\mathbf{m}_{r}, \bar{\phi}(t), t\right)\right| p_{0}\left(\mathbf{x}+\mathbf{m}_{r}, t\right) d \mathbf{x} \\
& \leq \int_{\mathbb{R}_{+}^{m}} c_{r}(\mathbf{x}, t) \Delta \hat{\phi} p_{0}\left(\mathbf{x}+\mathbf{m}_{r}, t\right) d \mathbf{x} \leq C_{r}(t) \Delta \hat{\phi},
\end{aligned}
$$

with $\left|\Delta \phi_{j}(\mathbf{x}, t)\right| \leq \Delta \hat{\phi}$ for all $j$ and some positive $c_{r}$ and $C_{r}$. The equation for $\bar{\phi}$ is then derived from (29) and after discarding small terms depending on $\Delta \hat{\phi}$ according to (36) we have

$$
\frac{d \bar{\phi}_{k}}{d t}=-\sum_{r=1}^{R} n_{r k} \int_{\mathbb{R}_{+}^{m}} w_{r}\left(\mathbf{x}+\mathbf{m}_{r}, \bar{\phi}(t), t\right) p_{0}\left(\mathbf{x}+\mathbf{m}_{r}, t\right) d \mathbf{x} .
$$

The equation for $p_{0}$ derived from the master equation is as in (15). Replace $\phi$ by $\bar{\phi}$ in (15) and ignore $\rho_{0}$. The difference between the right hand sides due to 
this change is denoted by $\rho_{r \bar{\phi}}$. With the assumption in Lemma 3.1 on $w_{r}$, there is a bound on $\rho_{r \bar{\phi}}$ which is linear in $\Delta \hat{\phi}$ when $\Delta \hat{\phi}$ is sufficiently small, see (36). The equation for $p_{0}$ and one reaction is now

$$
\begin{aligned}
\frac{\partial p_{0}(\mathbf{x}, t)}{\partial t} & =w_{r}\left(\mathbf{x}+\mathbf{m}_{r}, \boldsymbol{\phi}\left(\mathbf{x}+\mathbf{m}_{r}, t\right), t\right) p_{0}\left(\mathbf{x}+\mathbf{m}_{r}, t\right)-w_{r}(\mathbf{x}, \boldsymbol{\phi}(\mathbf{x}, t), t) p_{0}(\mathbf{x}, t) \\
& =w_{r}\left(\mathbf{x}+\mathbf{m}_{r}, \bar{\phi}(t), t\right) p_{0}\left(\mathbf{x}+\mathbf{m}_{r}, t\right)-w_{r}(\mathbf{x}, \bar{\phi}(t), t) p_{0}(\mathbf{x}, t)+\rho_{r \bar{\phi}}
\end{aligned}
$$

The FP equation derived from (38) is

$$
\frac{\partial p_{0}(\mathbf{x}, t)}{\partial t}=\left\{\sum_{i=1}^{m} m_{r i} \frac{\partial \bar{q}_{r 0}(\mathbf{x}, t)}{\partial x_{i}}+\sum_{i=1}^{m} \sum_{j=1}^{m} \frac{m_{r i} m_{r j}}{2} \frac{\partial^{2} \bar{q}_{r 0}(\mathbf{x}, t)}{\partial x_{i} \partial x_{j}}\right\}+\rho_{\bar{\phi}},
$$

with $\bar{q}_{r 0}(\mathbf{x}, t)=w_{r}(\mathbf{x}, \bar{\phi}(t), t) p_{0}(\mathbf{x}, t)$ and $\rho_{\bar{\phi}}=\sum_{r} \rho_{r \bar{\phi}}$. Neglecting the term $\rho_{\bar{\phi}}$, the FP equation for $p_{0}(\mathbf{x}, t)$ in conservation form is

$$
\frac{\partial p_{0}(\mathbf{x}, t)}{\partial t}=\sum_{r=1}^{R} \nabla \cdot \overline{\mathbf{F}}_{r 0}
$$

with the flux functions

$$
\overline{\mathbf{F}}_{r 0 i}=m_{r i}\left(\bar{q}_{r 0}+0.5 \mathbf{m}_{r} \cdot \nabla \bar{q}_{r 0}\right), i=1 \ldots m, r=1 \ldots R .
$$

The time evolution of the expected values $\overline{\boldsymbol{\phi}}(t)$ and the PDF $p_{0}(\mathbf{x}, t)$ is governed by a system of usually nonlinear IDEs (37) and a PDE (40). The differences between the solutions of (29) and (37) and the solutions of (39) and (40) are estimated in the next theorem. These differences are small if $\Delta \hat{\phi}$ is small.

Theorem 3.4. Assume that $w_{r}, r=1 \ldots R$, is bounded and has bounded first and second derivatives in $\mathbf{x}$ in $Q_{\eta} \times[0, T]$ and that $M(\mathbf{x}, t)=\sum_{r} w_{r} \mathbf{m}_{r} \mathbf{m}_{r}^{T} \in \mathbb{R}^{m \times m}$ is positive definite.

Let $\tilde{\boldsymbol{\phi}}$ be the solution of (29) and $\overline{\boldsymbol{\phi}}$ the bounded solution of (37) with identical initial conditions at $t=0$. Assume that $p_{0}$ is a PDF and that $\left|\phi_{k}(\mathbf{x}, t)-\bar{\phi}_{k}(t)\right| \leq$ $\Delta \hat{\phi}$ for all $k, \mathbf{x}$, and $t$. Then for $t \in[0, T]$

$$
\left|\tilde{\phi}_{k}(t)-\bar{\phi}_{k}(t)\right| \leq \Delta \hat{\phi} \sum_{r}\left|n_{r k}\right| \int_{0}^{t} C_{r}(\tau) d \tau \text {. }
$$

Let $\tilde{p}_{0}$ be the weak solution of (39) and $p_{0}$ the weak solution of (40) with the boundary conditions $\tilde{p}_{0}(\mathbf{x}, t)=0$ and $p_{0}(\mathbf{x}, t)=0$ on the boundary $\partial Q_{\eta}$ and identical initial conditions at $t=0$. Then for $\Delta \hat{\phi}$ sufficiently small and $t \in[0, T]$

$$
\left|\tilde{p}_{0}(\mathbf{x}, t)-p_{0}(\mathbf{x}, t)\right| \leq C_{\phi} \Delta \hat{\phi},
$$


where $C_{\phi}$ depends on $\eta, T, w_{r}, \bar{\phi}, m$, and $\mathbf{m}_{r}$.

Proof. The difference $\delta \phi_{k}=\tilde{\phi}_{k}-\bar{\phi}_{k}$ satisfies

$$
-\sum_{r}\left|n_{r k}\right| C_{r}(t) \Delta \hat{\phi} \leq \frac{d \delta \phi_{k}}{d t} \leq \sum_{r}\left|n_{r k}\right| C_{r}(t) \Delta \hat{\phi}, k=1 \ldots n,
$$

by (36). The bound on $\left|\delta \phi_{k}(t)\right|$ is obtained by Gronwall's lemma.

The difference $\delta p_{0}=\tilde{p}_{0}-p_{0}$ satisfies (40) with the source term $g=\rho_{\bar{\phi}}$. The boundary and initial condition is $\delta p_{0}=0$. Then $\delta p_{0}$ is a solution to (23) with $g$ added to the right hand side. As in the proof of Theorem 3.2, the maximum principle in Theorem 1 of [1] is applicable with $g$ bounded by a sum over $r$ of the bound in (36) and the bound on $\delta p_{0}$ follows without any smoothness assumptions on $\bar{\phi}$.

In $[15,17]$, the purpose of the splitting of the species is to have one set of slow variables and one set of fast variables. The fast variables correspond to $\bar{\phi}$ in (37) and the equation for the slow variables is the master equation corresponding to (40).

The equations in QCMD [7] also have a structure similar to (37) and (40). There is a system of equations in QCMD for the positions of the particles with an averaged right hand side as in (37) and there is a PDE for the wave function corresponding to (40) for the PDF $p_{0}$ here.

The case when all species are treated in the same manner without any assumption on the PDF as in (9) and $n=0$ results in the FP equation (4) or (5). The solution depends on time and the $m$-dimensional space and since $m=N$ no reduction of the original problem is achieved.

In the other extreme case $m=0$ and $p_{0}$ and $w_{r}$ depend only on $t$. The expected value in (14) also only depends on $t$ and $\phi_{k}$ equals $p_{k}$ apart from a scaling factor. The equation (22) agrees with (32) with $\boldsymbol{\psi}=\emptyset$. The macroscopic reaction rate equations for $\phi_{k}$ are the governing equations.

By replacing the integrals over $\mathbf{y}$ in (15) and (20) by summation over a $\mathbf{y}$ taking integer values, differential-difference equations of the same type as the master equation are obtained corresponding to (17) and (22). Summation over $\mathbf{x} \in \mathbb{Z}_{+}^{n}$ in (37) and over all reactions in (38) yields the equations in discrete space corresponding to (37) and (40).

If it is impossible to reduce $m$ to, say, 4 or 5 so that (40) can be solved numerically as a PDE, then $p_{0}$ in (37) can be generated by a stochastic algorithm such as in [13] without the exponential growth in $m$ of the computational work. This is a way of coupling stochastic simulation of some species while the expected values of the remaining species are given by IDEs.

Two different models mixing the macroscopic and the mesoscopic view have been derived:

1. (17) and (22), 
2. (37) and (40).

In both cases, the spatial dimension of the PDE is reduced from $m+n$ in the FP equation to $m$. The number of independent variables has increased from 1 to $n+1$ but this is much less of a problem from a computational point of view. The molecular species where the mesoscopic model is critical for an accurate description of the reactions should be among the selected $m$ compounds in the first group satisfying an FP equation. The expected values of the remaining $n$ species are solutions of $n$ additional equations. Assuming that the first $m$ species have the same type of probability distribution as the remaining $n$ species, we revert to the macroscopic reaction rate equations.

\section{Numerical results}

Consider the following nine reactions for the molecular species $X$ and $Y$ modeling the creation of two metabolites controled by two enzymes $E_{X}$ and $E_{Y}$ from [9]:

$$
\begin{array}{ll}
\emptyset \stackrel{w_{1}}{\longrightarrow} X & \emptyset \stackrel{w_{2}}{\longrightarrow} Y \\
X+Y \stackrel{w_{3}}{\longrightarrow} \emptyset & \\
X \stackrel{w_{4}}{\longrightarrow} \emptyset & Y \stackrel{w_{5}}{\longrightarrow} \emptyset \\
\emptyset \stackrel{w_{6}}{\longrightarrow} E_{X} & \emptyset \stackrel{w_{7}}{\longrightarrow} E_{Y} \\
E_{X} \stackrel{w_{8}}{\longrightarrow} \emptyset & E_{Y} \stackrel{w_{9}}{\longrightarrow} \emptyset
\end{array}
$$

The difference between the states and the propensities for the nine reactions are with $\mathbf{x}^{T}=(x, y)$ and $\mathbf{y}^{T}=\left(e_{X}, e_{Y}\right)$

$$
\begin{array}{llll}
\mathbf{n}_{1}^{T}=(-1,0,0,0), & w_{1}=\frac{k_{X} e_{X}}{1+\frac{x}{K_{I}}}, & \mathbf{n}_{2}^{T}=(0,-1,0,0), & w_{2}=\frac{k_{Y} e_{Y}}{1+\frac{y}{K_{I}}}, \\
\mathbf{n}_{3}^{T}=(1,1,0,0), & w_{3}=k_{2} x y, & \mathbf{n}_{4}^{T}=(1,0,0,0), & w_{4}=\mu x, \\
\mathbf{n}_{5}^{T}=(0,1,0,0), & w_{5}=\mu y, & \mathbf{n}_{6}^{T}=(0,0,-1,0), & w_{6}=\frac{k_{E_{X}}}{1+\frac{x}{K_{R}}}, \\
\mathbf{n}_{7}^{T}=(0,0,0,-1), & w_{7}=\frac{k_{E_{Y}}}{1+\frac{y}{K_{R}}}, & \mathbf{n}_{8}^{T}=(0,0,1,0), & w_{8}=\mu e_{X}, \\
\mathbf{n}_{9}^{T}=(0,0,0,1), & w_{9}=\mu e_{Y} . & &
\end{array}
$$

In the experiments, the coefficients are $k_{X}=k_{Y}=0.3, k_{2}=0.001, K_{I}=60, K_{R}=$ $30, k_{E_{X}}=k_{E_{Y}}=0.02$, and $\mu=0.002$.

The system with $m=2$ and $n=2$ is simulated with the FP equation using three different alternatives:

1. $X$ and $Y$ are stochastic variables, $E_{X}$ and $E_{Y}$ have normal distributions with a small $\sigma$ as in $(9) \Rightarrow$ three PDEs for $p_{0}(x, y, t)(17)$ and $p_{1}(x, y, t)$ and $p_{2}(x, y, t)(22)$ in $2 \mathrm{D}$,

2. $X$ and $Y$ are stochastic variables, $E_{X}$ and $E_{Y}$ have normal distributions with a small $\sigma$ and the expected values of $E_{X}$ and $E_{Y}$ are independent of 
$x$ and $y \Rightarrow$ one PDE for $p_{0}(x, y, t)(40)$ in 2D and two IDEs for $\bar{\phi}_{1}(t)$ and $\bar{\phi}_{2}(t)(37)$,

3. $X, Y, E_{X}, E_{Y}$ all have normal distributions with a small $\sigma \Rightarrow$ four reaction rate equations (2) or (32), (35).

The PDEs are discretized in an $m$-dimensional space by a finite volume method of second order and in time by the backward differentiation formula of order two (BDF-2) as in [11, 20]. Spatial first derivatives are approximated by an upwind scheme for improved spatial stability and BDF-2 is suitable for parabolic equations with its unconditional temporal stability. The system of nonlinear equations in every time step is solved by a Newton-Krylov method with GMRES [19]. The computational domain is the square $\Omega=\{0 \leq x, y \leq 200\}$.

The initial distribution of $p_{0}$ at $t=0$ is a Gauss pulse with center at $x=$ $y=60$. The solutions at $t=0,120$, and 3000, are displayed in Fig. 1 . The computed $p_{0}$ with Alternative 1 is close to the solution with Alternative 2 in the figure. The trajectory of the macroscopic solution with Alternative 3 is close to the trajectories of the peak of $p_{0}$. The difference between the solutions of $p_{0}$ at $t=3000$ is found in Fig. 2.a. It is small compared to the maximum of $p_{0}$ (see Fig. 3). The computed $\phi_{1}(\mathbf{x}, 3000)$ and $\phi_{2}(\mathbf{x}, 3000)$ only mildly dependent on $\mathbf{x}$ in Figs. 2.b and 2.c and $\phi_{1}(x, y, 3000)=\phi_{2}(y, x, 3000)$.

The time evolution of the maxima and the mean values of $p_{0}$ determined with $\phi(\mathbf{x}, t)$ and $\bar{\phi}(t)$ are displayed in Fig. 3. The mean value is constant because of the conservation of $p_{0}$. The difference is small between Alternatives 1 and 2 for this chemical system since $\phi(\mathbf{x}, t)$ is almost constant in $\mathbf{x}$ (see Figs. 2.b and 2.c). Alternative 2 is less computationally expensive and is the preferred model in this case.

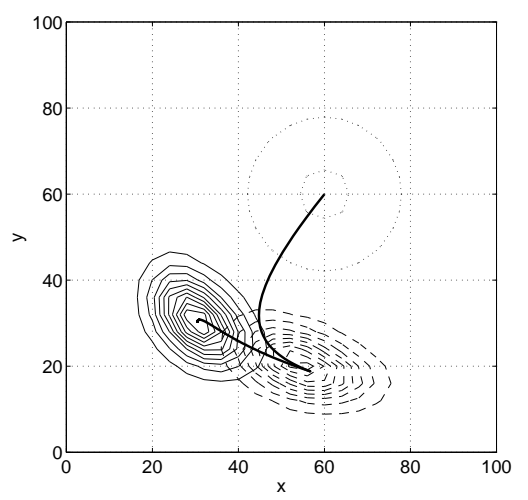

(a)

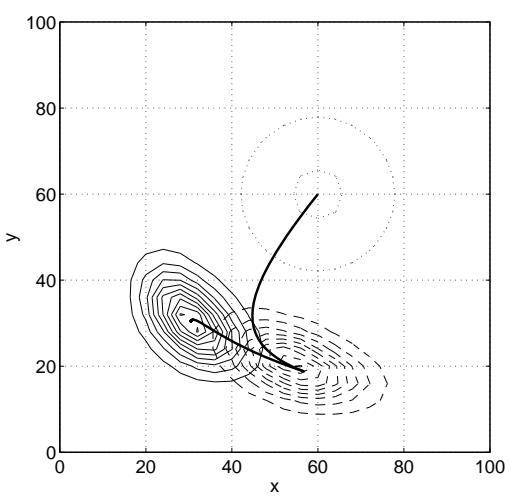

(b)

Figure 1: The isolines of $p_{0}$ are displayed at $t=0,120,3000$, using $\boldsymbol{\phi}(\mathbf{x}, t)$ (a) and $\bar{\phi}(t)(\mathrm{b})$. The solid thick line is the trajectory of the macroscopic solution. 


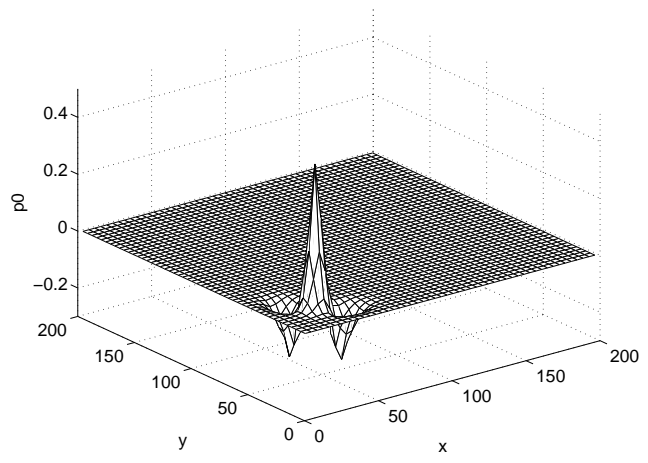

(a)

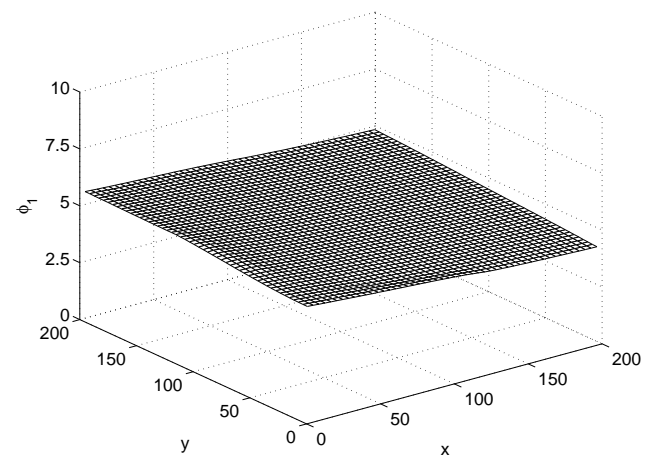

(b)

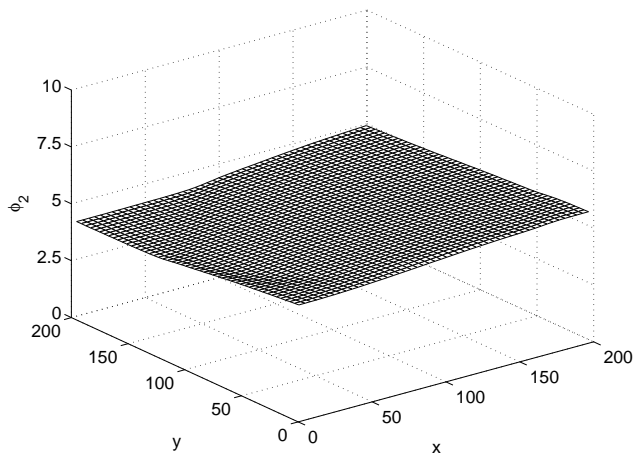

(c)

Figure 2: The difference between $p_{0}$ obtained with $\phi(\mathbf{x}, t)$ and $\bar{\phi}(t)$ at $t=3000$ (a), $\phi_{1}(\mathbf{x}, 3000)(\mathrm{b})$, and $\phi_{2}(\mathbf{x}, 3000)(\mathrm{c})$.

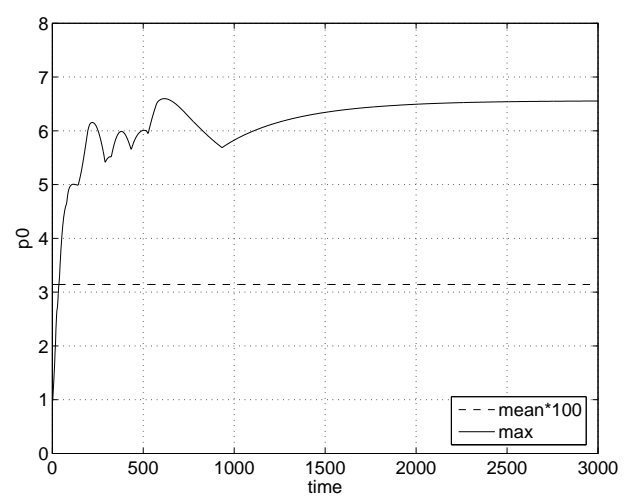

(a)

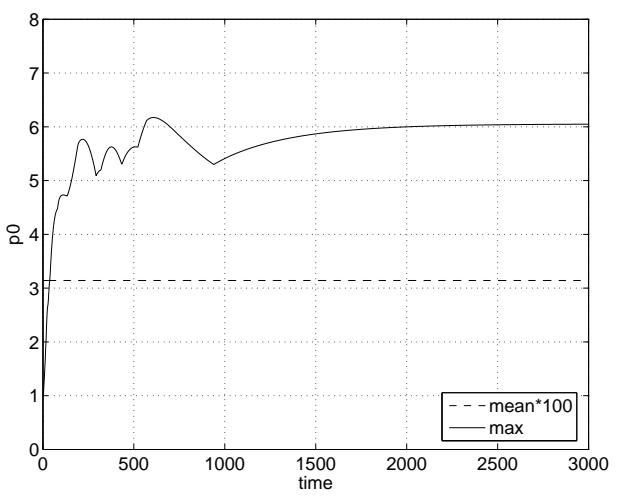

(b)

Figure 3: The maximum and the mean value of $p_{0}$ obtained with $\phi(\mathbf{x}, t)(\mathrm{a})$ and $\bar{\phi}(t)(\mathrm{b})$.

The solution of the macroscopic reaction rate equations is found in Fig. 4.a. The solution is compared to $\bar{\phi}_{1}(t)$ and $\bar{\phi}_{2}(t)$ from Alternative 2 in Fig. 4.b. The 
difference is barely visible in the plot. The mean values of $\boldsymbol{\phi}(\mathbf{x}, t)$ in Alternative 1 are $\left(\phi_{1}, \phi_{2}\right)=(7.98,4.93)$ at $t=120$ and $\left(\phi_{1}, \phi_{2}\right)=(4.96,4.96)$ at $t=3000$, also in good agreement with Fig. 4.b. The reaction rate equations yield good approximations of the expected values here.

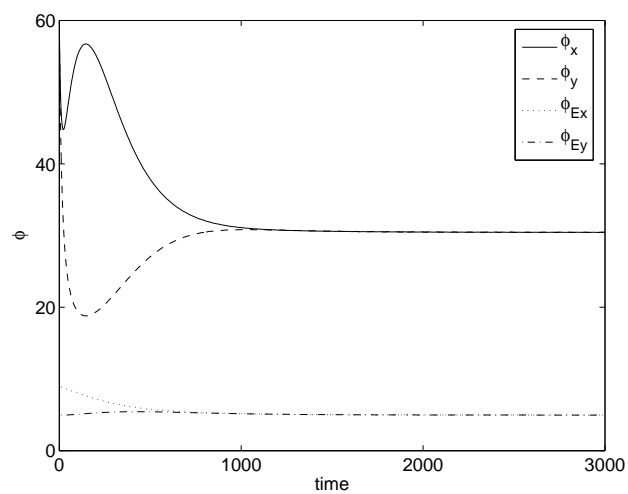

(a)

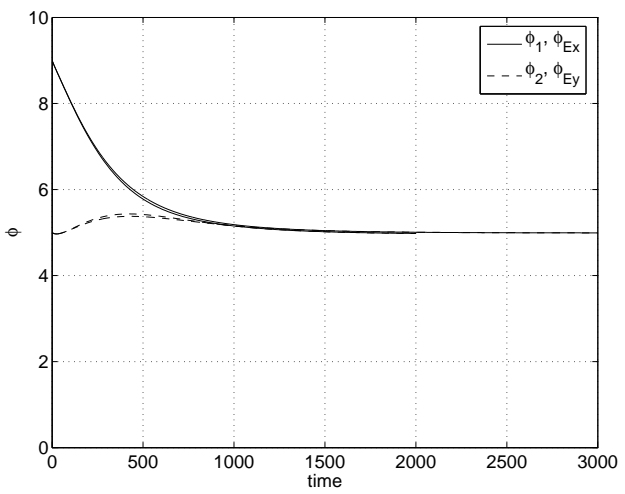

(b)

Figure 4: The solution of the reaction rate equations (a) and $\bar{\phi}_{1}(t)$ and $\bar{\phi}_{2}(t)$ compared with $\phi_{E_{X}}$ and $\phi_{E_{Y}}$ from the reaction rate equations (b).

\section{Conclusions}

Four different levels of modelling chemical reactions have been derived and discussed. The most complete model considered here is the mesoscopic master equation and the corresponding FP equation for the PDF of all molecular species (5). Assuming that the species can be divided into two sets where the species in the second set are independently and normally distributed, one FP equation is derived for the PDF of the first set of species (17) and equations for the partial first moments of the second set of species (22). If the variation of the conditional expected values in the second set is small over the first set, then a system of IDEs (37) and an FP equation (40) are the appropriate model equations. Finally, when also the species of the first set are independently and normally distributed, we obtain the macroscopic reaction rate equations (2). If there are $m$ species in the first set and $n$ in the second set, the spatial dimension of the FP equation is $m+n$. In the first simplification, $n+1$ PDEs are solved in $m$ dimensions. The equations of the second simplification are $n$ time-dependent IDEs and one PDE in $m$ dimensions. The reaction rate equations are a system of time-dependent ODEs for $m+n$ concentrations.

A four dimensional problem is reduced to two dimensions using (17), (22), and (37), (40), in a numerical example. These solutions are compared with the solution of the reaction rate equations. In this particular example, the difference between all the solutions is small, but this is in general not the case. 


\section{Acknowledgement}

This work has been supported by the Swedish Foundation for Strategic Research.

\section{Appendix}

The proofs of two lemmas in Sect. 3 are presented here.

Proof of Lemma 3.1.

Introduce the change of variables $z_{j}=\left(y_{j}-\phi_{j}\right) / \sigma$ in $E[w \mid \mathbf{x}]$ and let $\Delta \boldsymbol{\phi}=\sigma \mathbf{z}$ to obtain

$$
\begin{aligned}
E[w \mid \mathbf{x}]= & \gamma_{n} \sigma^{n} \int\left(w(\mathbf{x}, \boldsymbol{\phi}, t)+\sigma \sum_{j=1}^{n} c_{j}(\mathbf{x}, \boldsymbol{\phi}, t) z_{j}\right. \\
& +\rho(\mathbf{x}, \boldsymbol{\phi}, \sigma \mathbf{z}, t)) \exp \left(-0.5 \sum_{j=1}^{n} z_{j}^{2}\right) d \mathbf{z} \\
= & w(\mathbf{x}, \boldsymbol{\phi}, t)+\gamma_{n} \sigma^{n+1} \sum_{j=1}^{n} c_{j}(\mathbf{x}, \boldsymbol{\phi}, t) \int z_{j} \exp \left(-0.5 \sum_{j=1}^{n} z_{j}^{2}\right) d \mathbf{z} \\
& +(2 \pi)^{-n / 2} \int \rho(\mathbf{x}, \boldsymbol{\phi}, \sigma \mathbf{z}, t) \exp \left(-0.5 \sum_{j=1}^{n} z_{j}^{2}\right) d \mathbf{z} .
\end{aligned}
$$

The first integral in the expression for $E[w \mid \mathbf{x}]$ is zero and a bound on the second integral of $\rho, I_{\rho}$, is

$$
\begin{aligned}
\left|I_{\rho}\right| & =\left|\int \rho(\mathbf{x}, \boldsymbol{\phi}, \sigma \mathbf{z}, t) \exp \left(-0.5 \sum_{j=1}^{n} z_{j}^{2}\right) d \mathbf{z}\right| \\
& \leq \int \sum_{k=2}^{q} \sigma^{k} \sum_{|\boldsymbol{\delta}|=k} \xi_{\boldsymbol{\delta}}(\mathbf{x}, t) \prod_{j=1}^{n}\left|z_{j}\right|^{\delta_{j}} \exp \left(-0.5 z_{j}^{2}\right) d \mathbf{z} \\
& \leq 2^{n} \int_{\mathbb{R}_{+}^{n}} \sum_{k=2}^{q} \sigma^{k} \sum_{|\boldsymbol{\delta}|=k} \xi_{\boldsymbol{\delta}}(\mathbf{x}, t) \prod_{j=1}^{n} z_{j}^{\delta_{j}} \exp \left(-0.5 z_{j}^{2}\right) d \mathbf{z} \\
& =2^{n} \sum_{k=2}^{q} \sigma^{k} \sum_{|\boldsymbol{\delta}|=k} \xi_{\boldsymbol{\delta}}(\mathbf{x}, t) \prod_{j=1}^{n} \int_{0}^{\infty} z_{j}^{\delta_{j}} \exp \left(-0.5 z_{j}^{2}\right) d z_{j} .
\end{aligned}
$$

The integrals in the product all have bounds depending on $\delta_{j}$ and therefore on $k$ and $q$. There is a $\kappa_{1}(\mathbf{x}, t, n)$ such that

$$
(2 \pi)^{-n / 2}\left|I_{\rho}\right| \leq \sum_{k=2}^{q} \sigma^{k} \kappa_{1}=\kappa_{1} \sigma^{2}\left(1-\sigma^{q-1}\right) /(1-\sigma),
$$

The lemma is proved. 


\section{Proof of Lemma 3.3.}

The difference between $E[w]$ and the integral over $\mathbb{R}^{m}$ is

$$
\begin{aligned}
\delta I & =\gamma_{m} \int_{\mathbb{R}^{m} \backslash \mathbb{R}_{+}^{m}} w(\mathbf{x}+\mathbf{m}, t) \exp \left(-\sum_{j=1}^{m} \frac{\left(x_{j}+m_{r j}-\psi_{j}\right)^{2}}{2 \sigma^{2}}\right) d \mathbf{x} \\
& =\sigma^{m} \gamma_{m} \int_{\mathcal{U}} w(\boldsymbol{\psi}+\sigma \mathbf{z}, t) \exp \left(-0.5 \sum_{j=1}^{m} z_{j}^{2}\right) d \mathbf{z},
\end{aligned}
$$

with the change of variables $x_{j}=\sigma z_{j}-u_{j}, u_{j}=m_{r j}-\psi_{j}<0$, and $\mathcal{U}=$ $\bigcup_{j=1}^{m} U_{j}, U_{j}=\left\{\mathbf{z} \mid z_{j} \leq u_{j} / \sigma\right\}$. Then $\delta I$ is bounded by

$$
\begin{aligned}
|\delta I| & \leq \sigma^{m} \gamma_{m} \sum_{i=1}^{m} \int_{U_{i}}|w(\boldsymbol{\psi}+\sigma \mathbf{z}, t)| \exp \left(-0.5 \sum_{j=1}^{m} z_{j}^{2}\right) d \mathbf{z} \\
& \leq \sigma^{m} \gamma_{m} \sum_{i=1}^{m} \sum_{k=0}^{q} \sigma^{k} \sum_{|\boldsymbol{\delta}|=k} \eta_{\boldsymbol{\delta}}(\boldsymbol{\psi}, t) \int_{z_{i} \leq u_{i} / \sigma} \prod_{j=1}^{m}\left|z_{j}\right|^{\delta_{j}} \exp \left(-0.5 z_{j}^{2}\right) d \mathbf{z}
\end{aligned}
$$

The integrals can be written

$$
\begin{aligned}
& \int_{z_{i} \leq u_{i} / \sigma} \prod_{j=1}^{m}\left|z_{j}\right|^{\delta_{j}} \exp \left(-0.5 z_{j}^{2}\right) d \mathbf{z} \\
& =\int_{-\infty}^{u_{i} / \sigma}(-1)^{\delta_{i}} z_{i}^{\delta_{i}} \exp \left(-0.5 z_{i}^{2}\right) d z_{i} \prod_{j \neq i} \int\left|z_{j}\right|^{\delta_{j}} \exp \left(-0.5 z_{j}^{2}\right) d z_{j} .
\end{aligned}
$$

The product of the integrals has a bound depending on $\delta_{j}$ (cf. the proof of Lemma 3.1). A recursion formula applicable to the first integral is

$$
\begin{aligned}
& I_{k}=\int_{-\infty}^{v} z^{k} \exp \left(-0.5 z^{2}\right) d z=-v^{k-1} \exp \left(-0.5 v^{2}\right)+(k-1) I_{k-2}, k=2 \ldots, \\
& I_{1}=-\exp \left(-0.5 v^{2}\right), I_{0} \leq \int_{-\infty}^{v} \exp (-0.5 v z) d z=-2 v^{-1} \exp \left(-0.5 v^{2}\right) .
\end{aligned}
$$

Thus, the integrals in (42) are bounded by

$$
\mathcal{P}_{k-1}\left(u_{i} / \sigma\right) \exp \left(-0.5\left(u_{i} / \sigma\right)^{2}\right)
$$

where $\mathcal{P}_{k-1}$ is a polynomial of degree $k-1$. Sum all the terms in the estimate of $|\delta I|(42)$ and the lemma is proved.

\section{References}

[1] D. G. Aronson, J. Serrin, Local behavior of solutions of quasilinear parabolic equations, Arch. Rat. Mech. Anal., 25 (1967), p. 81-122. 
[2] F. Baras, M. M. Mansour, Microscopic simulations of chemical instabilities, Adv. Chem. Phys., 100 (1997), p. 393-474.

[3] H.-J. Bungartz, M. Griebel, Sparse grids, Acta Numerica, 2004, p. $147-269$.

[4] R. E. Caflish, Monte Carlo and quasi-Monte Carlo methods, Acta Numerica, 1998, p. 1-49.

[5] Y. Cao, D. Gillespie, L. Petzold, Multiscale stochastic simulation algorithm with stochastic partial equilibrium assumption for chemically reacting systems, J. Comput. Phys., 206 (2005), p. 395-411.

[6] P. G. Ciarlet, ed., Handbook of Numerical Analysis, Vol. X, Computational Chemistry, North-Holland, Amsterdam, 2003.

[7] P. Deuflhard, J. Hermans, B. Leimkuhler, A. E. Mark, S. ReICH, R. D. SkeEL, eds., Computational Molecular Dynamics: Challenges, Methods, Ideas, Lecture Notes in Computational Science and Engineering 4, Springer, Berlin, 1999.

[8] M. Ehrenberg, J. Elf, E. Aurell, R. Sandberg, J. Tegnér, Systems biology is taking off, Genome Res., 13 (2003), p. 2377-2380.

[9] J. Elf, P. LÖtstedt, P. SJöвerg, Problems of high dimension in molecular biology, in High-dimensional problems - $\mathrm{Nu}-$ merical treatment and applications, ed. W Hackbusch, Proceedings of the 19th GAMM-Seminar Leipzig 2003, p. 21-30, available at http://www.mis.mpg.de/conferences/gamm/2003/.

[10] J. Elf, J. Paulsson, O. G. Berg, M. Ehrenberg, Near-critical phenomena in intracellular metabolite pools, Biophys. J., 84 (2003), p. 154-170.

[11] L. Ferm, P. Lötstedt, P. SjöBerg, Adaptive, conservative solution of the Fokker-Planck equation in molecular biology, Technical report 2004-054, Dept of Information Technology, Uppsala University, Uppsala, Sweden, 2004, available at http://www.it.uu.se/research/reports/2004-054/.

[12] C. W. Gardiner, Handbook of Stochastic Methods, 2nd ed., Springer, Berlin, 2002.

[13] D. T. Gillespie, A general method for numerically simulating the stochastic time evolution of coupled chemical reactions, J. Comput. Phys., 22 (1976), p. $403-434$. 
[14] P. Guptasarma, Does replication-induced transcription regulate synthesis of the myriad low copy number proteins of Escherichia coli?, Bioessays, 17 (1995), p. 987-997.

[15] E. L. Haseltine, J. B. Rawlings, Approximate simulation of coupled fast and slow reactions for stochastic chemical kinetics, J. Chem. Phys., 117 (2002), p. 6959-6969.

[16] N. G. van Kampen, Stochastic Processes in Physics and Chemistry, NorthHolland, Amsterdam, 1992.

[17] T. R. Kiehl, R. M. Mattheyses, M. K. Simmons, Hybrid simulation of cellular behaviour, Bioinformatics, 20 (2004), p. 316-322.

[18] H. Kitano, Computational systems biology, Nature, 420 (2002), p. 206-210.

[19] D. A. Knoll, D. E. Keyes, Jacobian-free Newton-Krylov methods: a survey of approaches and applications, J. Comput. Phys., 193 (2004), p. 357-397.

[20] P. Lötstedt, S. Söderberg, A. Ramage, L. HemmingssonFRÄNDÉn, Implicit solution of hyperbolic equations with space-time adaptivity, BIT, 42 (2002), p. 134-158.

[21] C. V. Rao, A. P. Arkin, Stochastic chemical kinetics and the quasisteady-state assumption: Application to the Gillespie algorithm, J. Chem. Phys., 118 (2003), p. 4999-5010.

[22] M. Renardy, R. C. Rogers, An Introduction to Partial Differential Equations, Springer, New York, 1993.

[23] H. Risken, The Fokker-Planck Equation, 2nd ed., Springer, Berlin, 1996.

[24] M. Thattai, A. Van OudenaArden, Intrinsic noise in gene regulatory networks, Proc. Nat. Acad. Sci., 98 (2001), p. 8614-8619.

[25] J. M. G. Vilar, H. Y. Kueh, N. Barkai, S. Leibler, Mechanisms of noise-resistance in genetic oscillators, Proc. Nat. Acad. Sci., 99 (2002), p. 5988-5992. 